\title{
Unilateral Prefrontal Lesions Impair Memory-Guided Comparisons of Contralateral Visual Motion
}

\author{
Tㅜatiana Pasternak, Leo L. Lui, and Philip M. Spinelli \\ Department of Neurobiology and Anatomy, and Center for Visual Science, University of Rochester, Rochester, New York 14642
}

\begin{abstract}
The contribution of the lateral prefrontal cortex (LPFC) to working memory is the topic of active debate. On the one hand, it has been argued that the persistent delay activity in LPFC recorded during some working memory tasks is a reflection of sensory storage, the notion supported by some lesion studies. On the other hand, there is emerging evidence that the LPFC plays a key role in the maintenance of sensory information not by storing relevant visual signals but by allocating visual attention to such stimuli. In this study, we addressed this question by examining the effects of unilateral LPFC lesions during a working memory task requiring monkeys to compare directions of two moving stimuli, separated by a delay. The lesions resulted in impaired thresholds for contralesional stimuli at longer delays, and these deficits were most dramatic when the task required rapid reallocation of spatial attention. In addition, these effects were equally pronounced when the remembered stimuli were at threshold or moved coherently. The contralesional nature of the deficits points to the importance of the interactions between the LPFC and the motion processing neurons residing in extrastriate area MT. Delay-specificity of the deficit supports LPFC involvement in the maintenance stage of the comparison task. However, because this deficit was independent of stimulus features giving rise to the remembered direction and was most pronounced during rapid shifts of attention, its role is more likely to be attending and accessing the preserved motion signals rather than their storage.
\end{abstract}

Key words: cortical damage; direction discrimination; motion perception; prefrontal cortex; visual attention; working memory

\section{Introduction}

The role of prefrontal cortex (PFC) in working memory, the process of maintenance and manipulation of information during goal directed behaviors, has been debated for many years, beginning with the observation that monkeys with bilateral prefrontal lesions were unable to remember previously bated spatial locations (Jacobsen, 1936). Subsequent work revealed that this deficit could be erased by darkening the room during the delay, leading to the hypothesis of increased susceptibility to interference following prefrontal damage (Malmo, 1942; Bartus and Levere, 1977), and raising a possibility of this region playing a key role in attentional control, an idea that over the years received strong experimental support (for review, see Miller and Buschman, 2013). However, the discovery of persistent delay activity in the dorsolateral region of the PFC (Fuster and Alexander, 1971; Funahashi et al., 1989) revived the idea of the role for the PFC in

Received Dec. 27, 2014; revised Feb. 17, 2015; accepted March 26, 2015.

Author contributions: T.P. designed research; T.P., L.L.L., and P.M.S. performed research; T.P., L.L.L., and P.M.S. analyzed data; T.P. wrote the paper.

This work was supported by Grant R01 EY11749 (T.P.) and P30 EY01319 (Center for Visual Science), and L.L. was supported by the NHMRC of Australia (Project Grant 1066232), and the Australian Research Council (DECRA130100493, CE140100007, and SR1000006). We thank Ben Hayden, Krystel Huxlin, Brad Postle, and Kostas Michalopoulos for comments on the paper, Dr Richard Saunders for invaluable surgical assistance with one of the animals, and Tracy Bubel, Marty Gyra, and Johnny Wen for excellent technical assistance.

The authors declare no competing financial interests.

Correspondence should be addressed to Dr Tatiana Pasternak, Department of Neurobiology and Anatomy, Box 603, University of Rochester, Rochester, NY 14642. E-mail: tania@cvs.rochester.edu.

L.L. Lui's present address: Department of Physiology, Monash Vision Group, Australian Research Council Centre of Excellence for Integrative Brain Function, Monash University, Australia.

DOI:10.1523/JNEUROSCI.5265-14.2015

Copyright $\odot 2015$ the authors $\quad 0270-6474 / 15 / 357095-11 \$ 15.00 / 0$ working memory and led to the influential proposal that prefrontal cortex is the site of sensory storage (Goldman-Rakic, 1995). The finding of the "mnemonic scotoma" produced by dorsolateral PFC lesion (Funahashi et al., 1993) further cemented this view.

However, there is accumulating evidence provided by neuroimaging, physiological, and behavioral studies that argues against lateral PFC (LPFC) being the site of sensory storage during working memory tasks and suggest the involvement of sensory cortex in maintaining stimulus representations during such tasks (Curtis and D'Esposito, 2003; Pasternak and Greenlee, 2005; Tsujimoto and Postle, 2012; Sreenivasan et al., 2014; D'Esposito and Postle, 2015). A compelling challenge to persistent delay activity in the LPFC being a reflection of storage came from a study designed to separately examine representation of remembered and attended locations during a memory saccade task (Lebedev et al., 2004). The results revealed that the bulk of delay activity recorded during this task could be attributed to spatial attention, rather than to storage of remembered locations, the finding interpreted by the authors as evidence for the role of LPFC in topdown control of attention. Indeed, the idea that LPFC is important for directing attention to internal sensory representations and providing top-down influences to sensory neurons is widely accepted (Miller and Cohen, 2001; Miller and Buschman, 2013). Additional challenge to the idea of sensory storage residing in the PFC is the absence of persistent stimulus-selective delay activity during nonspatial working memory tasks (Zaksas and Pasternak, 2006; Hussar and Pasternak, 2012). Thus, the question of the role LPFC neurons play in sensory working memory is still unresolved. Are these neurons important for storage of sensory 
information, for directing attention to sensory representations stored elsewhere, or both?

In the present study, we addressed this question by examining the behavioral effects of permanent unilateral LPFC lesions during comparisons of two visual motion stimuli separated by a memory delay. The lesions included regions anatomically connected to the motion processing areas MT and MST (Barbas, 1988; Petrides and Pandya, 2006; Ninomiya et al., 2012) and shown to be active during all stages of memory-guided motion comparison tasks (Zaksas and Pasternak, 2006; Hussar and Pasternak, 2009, 2012, 2013).

The lesions impaired comparisons of motion directions within the hemifield contralateral to the lesioned hemisphere. This impairment was apparent only at longer memory delays, was independent of the type of stimulus signaling motion direction, and was most pronounced when the task required rapid reallocation of spatial attention. These results demonstrate a functional link between LPFC and the motion processing cortical neurons during working memory tasks and demonstrate the importance of the LPFC in the maintenance of the remembered motion. However, because this impairment did not depend on the type of motion giving rise to the remembered direction and was most affected during attentional shifts, this role is more likely to be related to accessing and attending to the preserved signals rather than their storage.

\section{Materials and Methods}

Subjects. We used four adult male macaque monkeys weighing $8-10 \mathrm{~kg}$. Before the placement of lesions in three of the animals (M123, M601, M908), extensive recordings from the lateral PFC were performed in all three animals. Results of these recordings have been reported previously (Zaksas and Pasternak, 2006; Hussar and Pasternak, 2009, 2010, 2012, 2013). An additional monkey (M202) served as a control. This animal had undergone identical behavioral training, but was not recorded from and received no lesion. All the experiments were performed in accordance with the guidelines published in the National Institutes of Health Guide for the Care and Use of Laboratory Animals (revised 1996) and were approved by the University of Rochester Committee for Animal Research.

Visual stimuli. The stimuli and the behavioral tasks were similar to those used in previous studies from this laboratory (Bisley and Pasternak, 2000; Bisley et al., 2001, 2004). Stimuli were presented on a video monitor (17 inch Nanao FlexScan T560i or 19 inch IIyama Vision Master Pro 513 , both running at $1152 \times 870$ pixel resolution and a $75 \mathrm{~Hz}$ refresh rate), placed $57 \mathrm{~cm}$ in front of the monkeys. They consisted of dots placed randomly in a circular aperture and having a constant translational step size $(\Delta x)$ and temporal interval $(\Delta t=13 \mathrm{~ms}$; Rudolph and Pasternak, 1999; Bisley and Pasternak, 2000; Bisley et al., 2004). The dots were $0.03^{\circ}$ of visual angle in diameter with a luminance of $15 \mathrm{~cd} / \mathrm{m}^{2}$, shown on a dark background of $0.1 \mathrm{~cd} / \mathrm{m}^{2}$. Each dot persisted for the entire duration of the stimulus. The dot density was $4.7 \mathrm{dots} /{ }^{\circ 2}$ and their speed was set to $5-15 \%$. On each video frame, the range of directions in the stimulus could be specified and each dot was assigned a direction chosen randomly from a specified distribution (Fig. 1A). The direction of motion for each dot was randomly chosen from a specified uniform distribution of directions. Two types of random-dot motion stimuli were used. Direction range stimuli (Fig. $1 A$, left and middle): in each frame, the dots moved independently in any direction chosen at random from a uniform distribution that could range from $0^{\circ}$ to $355^{\circ}$. In one version of the task, direction range was varied only during the first stimulus (S1). In the other version, direction range was varied only during the second stimulus (S2). Motion signal stimuli (Fig. 1A, right): in each frame, a set proportion of randomly selected dots (the percentage signal) moved coherently, while the others were displaced in random directions (Fig. $1 A$, right). The visual stimuli were placed at specified locations of both hemifields. In
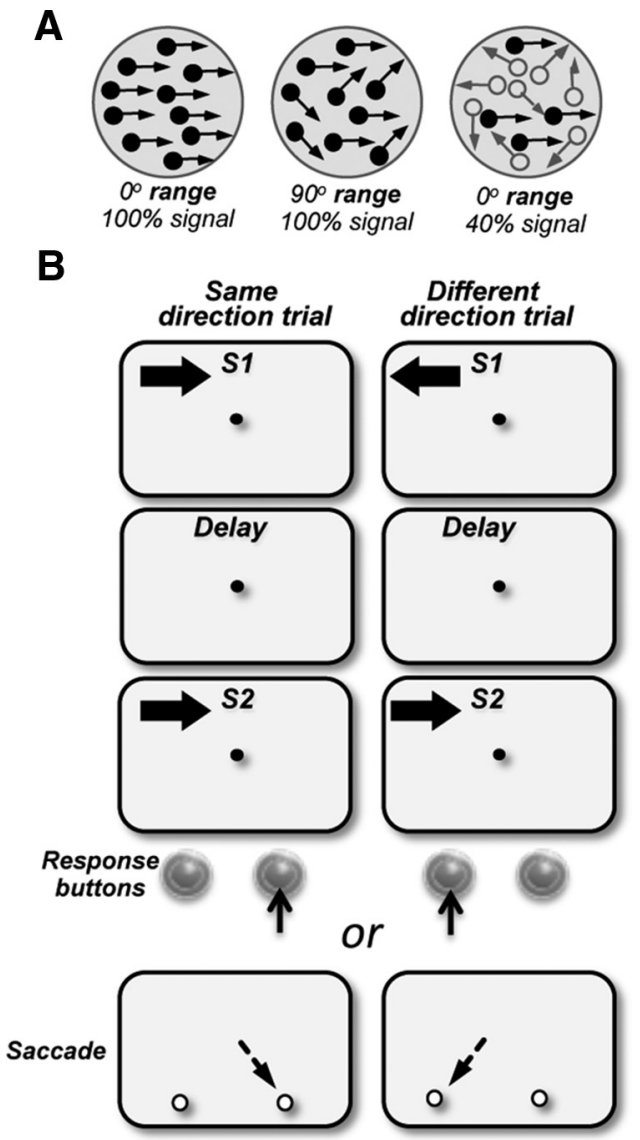

Figure 1. Visual stimuli and the behavioral task. $\boldsymbol{A}$, Direction range stimuli (left and middle dot fields): stimuli consisted of random dots displaced in directions chosen from a predetermined distribution. The width of the distribution of directions that determined the range of directions within which, individual dots moved was varied between $0^{\circ}$ (all dots moving in the same direction) and $360^{\circ}$ (dots moving in all directions). Motion signal thresholds (left and right dot fields): stimuli consisted of a proportion of dots moving in the same direction in a field of dots moving in random directions (signal dots, filled circles; noise dots, open circles). $\boldsymbol{B}$, Behavioral task: the animals maintained fixation on a target at the center of the display and reported whether the two stimuli, S1 and S2, separated by a delay moved in the same or in different directions by pressing one of the two response buttons (M123 and M601) or making a saccade to one of the two targets (M908, M202) which appeared after the offset of the fixation target. S1 and S 2 were $4^{\circ}-5^{\circ}$ diameter and moved at $10^{\circ}-15^{\circ} / \mathrm{s}$. In separate blocks of trials, stimuli were presented in the upper visual field on either side of the vertical meridian, in the ipsilesional and contralesional quadrants. On each trial, the direction of $\$ 1$ was chosen at random from a set of eight directions; S2 moved either in the same or opposite direction as S1.

monkeys with prefrontal lesions, these locations were selected based on psychophysical mapping performed at the start of behavioral training.

Behavioral tasks. The monkeys were trained to fixate a small spot at the center of the display while performing the discrimination task in a selected location of the visual field. The monkeys viewed a pair of $500 \mathrm{~ms}$ random dot stimuli, S1 and S2, moving either in the same or opposite directions. The two stimuli were separated by a delay that varied between 0.1 and $3 \mathrm{~s}$ in separate blocks of trials. Two of the monkeys (M123 and M601) reported whether the two directions were the "same" or "different" by pressing the right or left buttons, respectively, while the third animal (M908) reported its decision by making an eye movement to one of two saccade targets which appeared on the display side by side after the fixation point was turned off. Individual trials were separated by a $3 \mathrm{~s}$ delay. An incorrect response resulted in a 3-5 s tone and no reward. A break in fixation or a response during the trial resulted in a distinct $1-3 \mathrm{~s}$ tone and termination of the trial. A correct response was rewarded with a drop of fruit juice or water.

The direction of motion in S1 was varied on a trial-to-trial basis, and was picked randomly from a set of eight directions. The direction of S2 
was always set to the same or opposite direction to S1. The sequence of presentations of same/different stimuli was also randomized from trial to trial. To avoid positional biases, a correction procedure was used: after three consecutive errors on one button, the same trial was repeated until the animal made a correct response (which was not included in the data analysis).

Threshold measurements and data analysis. S1 and S2 stimuli either moved in the same direction or in opposite directions. The discrimination difficulty was changed by varying the range of directions within which the dots moved (direction range task) or by varying the proportion of the dots moving coherently (motion signal task). One of the two comparison stimuli was always coherent. Stimulus configurations used in each task are illustrated in individual data figures.

Thresholds were measured after the monkey reached criterion performance on the easiest level of the task (three consecutive sessions $\geq 90 \%$ correct or four consecutive sessions $\geq 80 \%$ correct). A staircase procedure was used to measure the threshold: three correct responses resulted in a less discriminable stimulus (e.g., a broader direction range), and each incorrect response decreased the difficulty of discrimination. Thresholds were estimated by fitting the data with a Weibull function, weighted by the number of trials at each point, using the maximum likelihood method (Quick, 1974). The thresholds were taken as the stimulus value corresponding to $75 \%$ correct. Psychophysical data were collected continuously for a period of 12-18 months.

The thresholds were measured to assess the effect of memory delay and of spatial uncertainty for stimuli presented in the ipsilesional and contralesional hemifields. When the length of the delay was manipulated, S1 and S2 appeared at the same spatial location, either contralesional or ipsilesional (Fig. 1B). We also used a direction comparison task in which S1 and S2 were spatially separated within the same hemifield. In one version of this task, the location of S1 and S2 were always highly predictable while in the other version, the location of S2 was uncertain. Under conditions of uncertainty, S2 could either appear at the same location as S1 or at a location $-16^{\circ}$ away in the same hemifield (see Fig. 7). An unpredictable location of the comparison stimulus requires rapid shifts spatial attention, a function that may be affected by prefrontal damage.

To measure range thresholds for a given delay condition, each testing session consisted of 200-300 trials. To obtain reliable data when the location of S2 was uncertain, because the thresholds were measured for each of the two S2 locations, it was necessary to increase the number of trials to 400-450 during these testing sessions.

Analysis of covariance (ANCOVA; Kutner et al., 2004) was used to evaluate changes in motion thresholds with delay for stimuli in the two hemifields. Stimulus location was used as a factor and delay as a covariate. The effect of uncertain S2 location within each hemifield for data pooled from the three animals was assessed with the Welch two-sample $t$ test. These analyses were performed using MATLAB (MathWorks). Paired $t$ tests were used to compare thresholds for the two hemifields for a single delay condition.

Lesion placement. Before lesion placement, extensive recordings from lateral prefrontal cortex were performed in all three animals. In two of the monkeys (M123, M601) the lesions were made with ibotenic acid injected into the prearcuate region in one of the hemispheres. In the third animal (M908), the damage was inadvertent, caused by an infection in the recording chamber positioned above the posterior PFC.

In the two animals that received chemical lesions, ibotenic acid (10 $\mu \mathrm{g} / \mu \mathrm{l}$ ) was injected at six sites anterior to the arcuate sulcus in one hemisphere. Injections were guided by magnetic resonance images taken before the surgery. Each injection $(1.5 \mu \mathrm{l})$ was made with a Hamilton syringe with $\sim 2 \mathrm{~mm}$ separation at several depths at each location. M601 received injections into the left hemisphere and the damage in M908 was in the right hemisphere. M123 received injections only into the right hemisphere. The location and the extent of damage were determined from subsequent histology. Behavioral testing began within a weeks of lesion placement.

Histology. At the conclusion of behavioral testing, the animals were deeply anesthetized with an overdose of barbiturate and perfused with a saline rinse followed by $4 \%$ paraformaldehyde fixative solution. Two rinses of $10 \%$ and $30 \%$ sucrose solution were used to remove excess fixative and to help cryoprotect the tissue. The brains were removed, blocked, and sectioned at a thickness of $50 \mu \mathrm{m}$ with a freezing microtome. Adjacent sections at intervals of $\sim 0.5 \mathrm{~mm}$ were stained for cytochrome oxidase (Wong-Riley, 1979) and with cresyl violet.

\section{Results}

\section{Lesion extent}

The damage was reconstructed from Nissl- and cytochrome oxydase-stained sections (Fig. 2). The lesion in M601 was relatively small, involving primarily areas $8 \mathrm{Av}$ and $8 \mathrm{Ad}$ in the right hemisphere. In monkey M908, the damage was also in the right hemisphere and involved the tissue around the posterior principal sulcus, the upper and lower limbs of the arcuate sulcus (FEF), small portions of ventral and dorsal regions of $46 / 9$ and areas $8 \mathrm{Ad}$ and $8 \mathrm{Av}$.

The damage in M123 was more extensive. In the right hemisphere, it included area $8 \mathrm{Av}$ and $8 \mathrm{Ad}$ and the most posterior portion of the principal sulcus (9/46d). The damage also extended into the dorsal premotor cortex, including area $6 \mathrm{~d}$. Histology also revealed unintended damage in the left hemisphere that most likely occurred during earlier recording experiments. This damage was limited to the dorsal convexity above the principal sulcus (area 9/46d) and a small most anterior portion of area $8 \mathrm{Ad}$. This unintended damage creates a problem with the definition of stimulus location as contralesional or ipsilesional. Because this damage was limited largely to the region unaffected in the other two monkeys and we found no detectible behavioral deficits in any of the tasks for stimuli contralateral to this damage, in the rest of paper we will refer to stimuli presented in right hemifield of this animal as ipsilesional and in the left hemifield as contralesional. Importantly for the goals of the present study, in all monkeys the damage included prearcuate regions that send direct projections to motion processing areas MT and MST from area $8 \mathrm{Av}$ and to parietal cortex from area $8 \mathrm{Ad}$ (Petrides and Pandya, 2006).

\section{Thresholds were unaffected at short delays}

To assess the effect of the PFC damage on sensory components of the task, thresholds were measured with the shortest possible delay (100-250 ms) between the two stimuli, S1 and S2. This condition allowed us to examine the process of motion integration with little demand placed on sensory storage. The direction range thresholds were measured by varying the direction range in $\mathrm{S} 1$, while $\mathrm{S} 2$ always contained coherent motion $\left(0^{\circ}\right.$ range). $\mathrm{S} 1$ and $\mathrm{S} 2$ moved in the same or opposite directions and always appeared at the same location. Thresholds for each location were measured in separate blocks of trials and we compared performance with stimuli presented in opposite hemifields at equal eccentricity (Fig. 3). The three lesioned monkeys showed no differences in thresholds between the two hemifields (Fig. $3 B$; M123, $p=0.35$; M601, $p=0.64$; M908, $p=0.47$; two-tailed $t$ test). Similarly, in the nonlesioned monkey (M202), the range thresholds measured for the right and left hemifields were largely equivalent $(p=0.39$; two-tailed $t$ test). The similarity of range thresholds for ipsilesional and contralesional stimuli measured at the shortest delay suggests that the process of motion integration, likely to be performed by MT neurons (Bisley et al., 2004), is unlikely to be influenced by the top-down influences arriving from the PFC. This result differs from the effects of a unilateral MT/MST lesion on range thresholds measured during an identical behavioral task (Rudolph and Pasternak, 1999). In that study, range thresholds for contralesional stimuli were affected even at the shortest delay, highlighting the importance of MT for motion integration (Movshon et al., 1985; Bisley et al., 2004). 

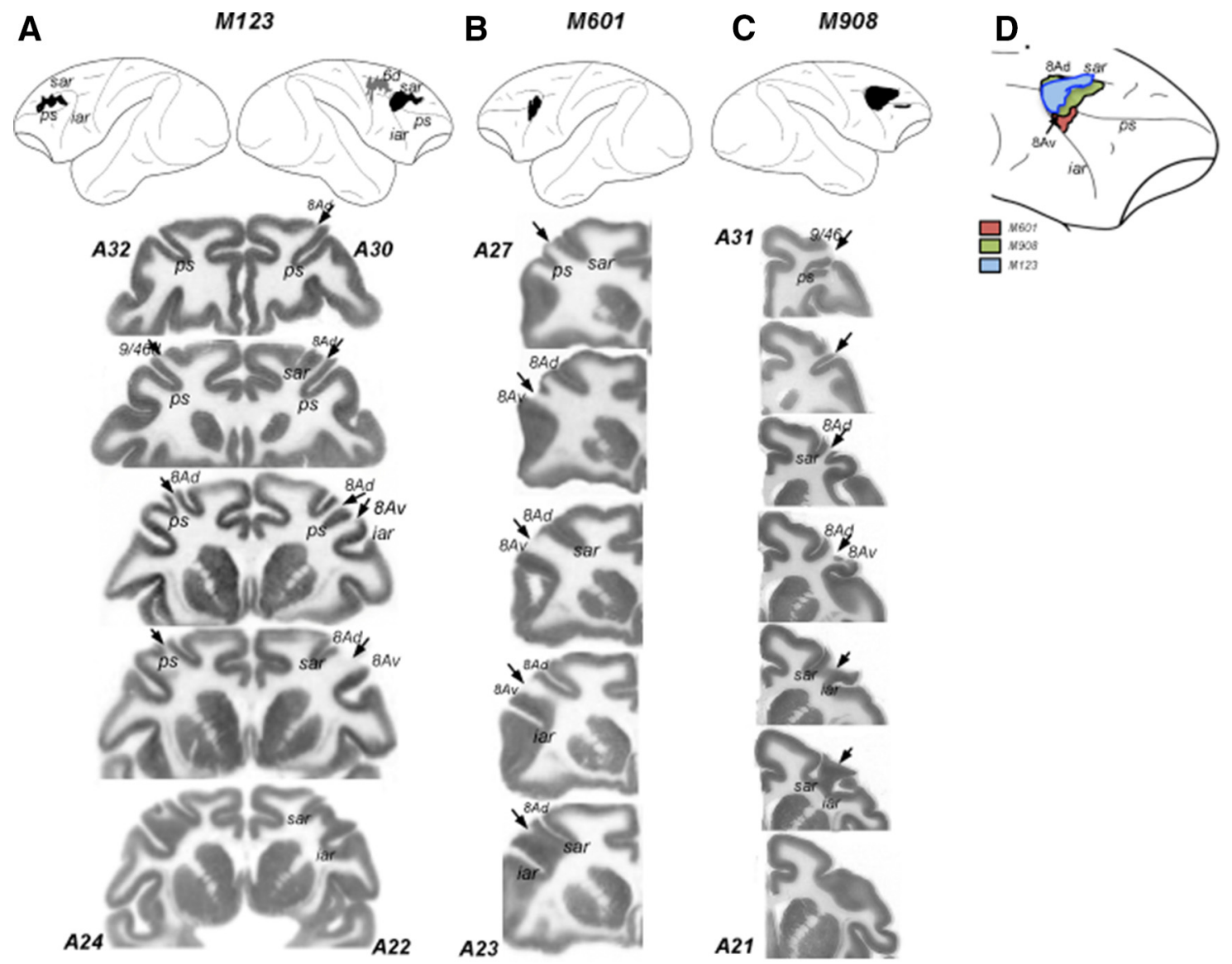

Figure 2. Lesion reconstructions. Top, Damage from both surgical lesions and unintended damage are shown for all three animals; these have been reconstructed from histological sections and projected onto a lateral view of the macaque brain. Both hemispheres are shown for M123 (A); only the hemispheres with damage are shown for M601 (B) and M908 (C). Black areas indicate areas of damage to LPFC, and gray areas indicate damage to the dorsal premotor cortex. Numbers correspond to anteroposterior (AP) distance (mm) from the interaural plane. The lines delineate the region represented in the coronal sections shown below. Bottom, Coronal sections/slices showing damage. $\boldsymbol{A}-\boldsymbol{C}$, Cytochrome oxidase stained histological sections showing the damage in in the three animals. Discontinuities in the gray matter are the damaged regions devoid of neurons and indicated by the arrows. Numbers correspond to AP distance from the interaural plane. For M123, the two hemispheres shown in a single section are in slightly different AP coordinates. The estimates of AP coordinates are based on the Paxinos et al. (2000) stereotaxic atlas. D, Comparison of lesions in the three monkeys shown on the lateral view of the right hemisphere. The lesions were transferred onto the same hemisphere to visualize the extent of overlap between the damage in individual animals. The diagram only shows the lesions in the prearcuate region. ps, Principal sulcus; sar, superior arcuate; iar, inferior arcuate.

\section{Deficits in motion thresholds increase with memory delay}

Previous work showed that motion integration thresholds, like other measures of discrimination abilities, worsen with the length of the delay interposed between the remembered and the current comparison stimuli, likely indicative of the rate of decay of signals temporarily stored for later retrieval (Bisley and Pasternak, 2000; Pasternak and Greenlee, 2005). We examined the effects of the LPFC damage on the rate of information decay (Fig. 3C). To facilitate the comparison between animals and to estimate the time course of degradation, individual thresholds were recomputed relative to thresholds measured at the shortest delay and fit by an exponential. In all monkeys, the performance decreased with delay.

An ANCOVA with location and delay as a covariates, revealed highly significant effect of delay on thresholds $\left(F_{(1,72)}=127.6\right.$; $\left.p=2.6 \mathrm{e}^{-16}\right)$ as well significant effect of location $\left(F_{(1,70)}=32.3\right.$; $p=2.6 \mathrm{e}^{-07}$ ), indicating that the performance was worse at longer delays and with contralesional stimuli. This analysis also revealed a significant interaction between the delay and stimulus location $\left(F_{(1,72)}=19.3 ; p=3.7 \mathrm{e}^{-05}\right)$, suggesting that the effect of delay was significant more pronounced for contralesional stimuli. The same analysis of the intact monkey (M202) data revealed the effect of delay $\left(F_{(1,21)}=15.3 ; p=0.0008\right)$, but no effect of location $\left(F_{(1,21)}=0.4 ; p=0.53\right)$, and no interaction between location and delay $\left(F_{(1,21)}=0.26 ; p=0.61\right)$. For the monkeys with damage, the asymmetries between thresholds measured in the two locations were characterized by faster deterioration of contralesional thresholds with increasing delay. This was also apparent in different slopes of the exponentials used to fit the data; this was confirmed by significant interaction effect between delay and location on the log-transformed thresholds $\left(F_{(1,72)}=\right.$ $\left.19 ; p=3.7 \mathrm{e}^{-05}\right)$.

The exponential functions fitted to the combined and individual data provided estimates of the length of time the animals were able to reliably retain and use motion direction. The loss of thresholds with time was most pronounced in M908, the monkey with the largest lesion (tau: $15.9 \mathrm{~s}$ (ipsi), $8.6 \mathrm{~s}$ (contra); $p=$ 0.0008 ; Figure $3 D$, summary). However, the loss with delay was also quite rapid in monkey M601, the animal with the smallest lesion, limited largely to area $8 \mathrm{~A}$ (tau: $13.7 \mathrm{~s}$ (ipsi), $8.8 \mathrm{~s}$ (contra); $p=0.015$ ). Interestingly, in the animal with the more extensive damage (M123), the slopes of the functions relating thresholds to delay for the two hemifields were more similar and the interaction effect failed to reach significance (tau: $16.7 \mathrm{~s}$ (ipsi), 11.6 $s($ contra $) ; p=0.1)$. In that monkey the cortical damage spared 
A

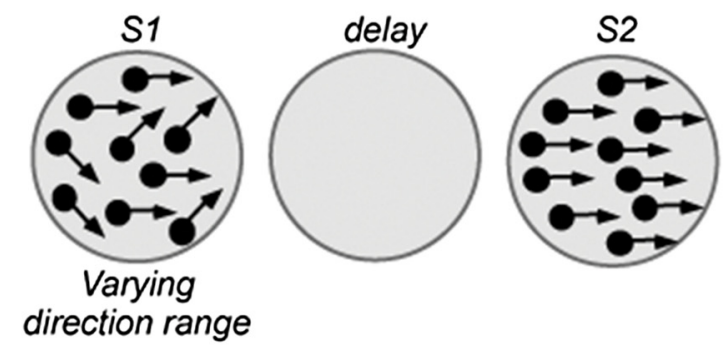

B
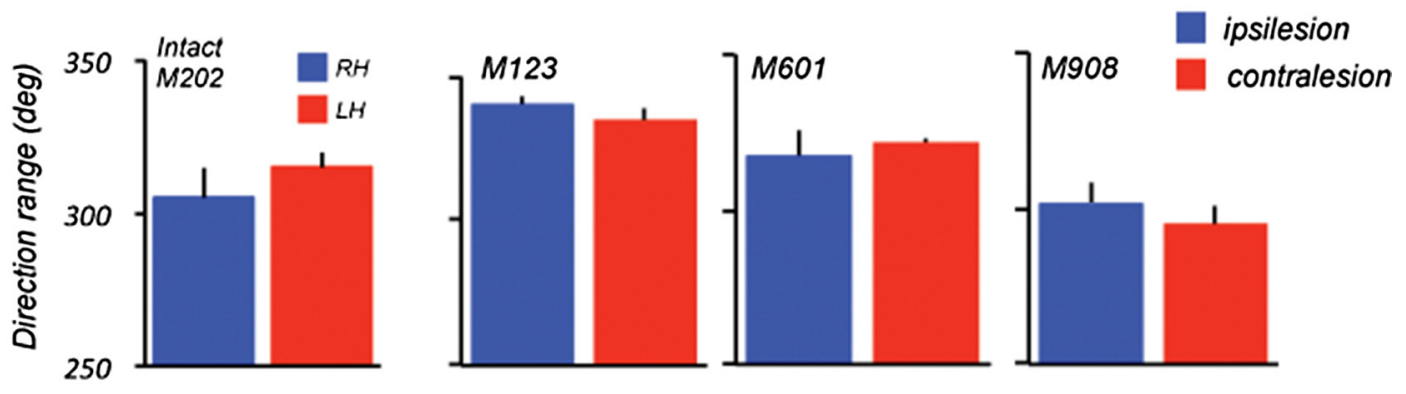

C
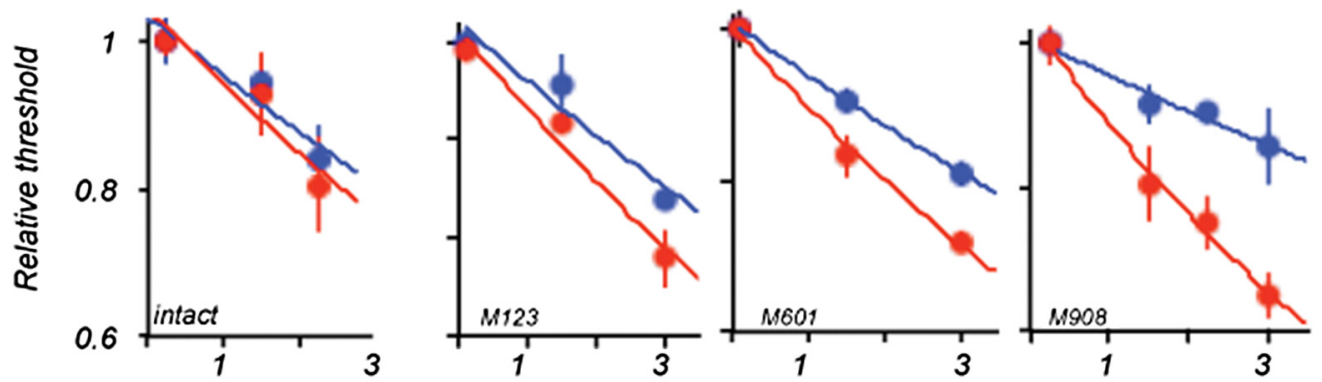

D

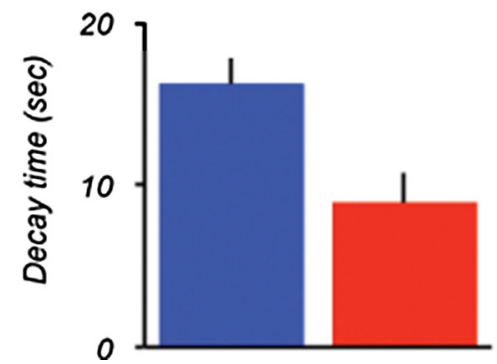

Delay (sec)

Figure 3. Lesion effects on direction range thresholds: $S 2$ at $0^{\circ}$ range. $A$, Stimulus configuration during the task. Thresholds were measured by varying direction range during $S 1$. The comparison S2 always moved coherently. The stimuli were placed in the following locations: M123 and M601, $\pm 8^{\circ}$ from VM, $2^{\circ}$ above HM; M908 and M202 (intact), $\pm 5^{\circ}$ from VM, $5^{\circ}$ above HM. $B$, Range thresholds measured at short delays (100 ms delay for M123 and M601; $250 \mathrm{~ms}$ delay for M908 and the intact animal M202). There was no difference between ipsilesional and contralesional thresholds for the three animals (M123, $p>0.3 ; \mathrm{M} 601, p>0.6 ; \mathrm{M} 908, p>0.4, t$ test). The thresholds for the intact monkey were also similar for the two hemifields ( $p>0.4, t$ test). $C$, Effect of delay on range thresholds in the two hemifields. For each animal, individual thresholds were normalized relative to thresholds measured at the shortest delay and fitted with an exponential function. The data were analyzed with the ANCOVA (see Materials and Methods). $\boldsymbol{D}$, Comparison of average decay times (tau) computed by fitting an exponential to the ipsilesional and contralesional data for the three lesioned monkeys. The decay time was shorter for the contralesional dataset (ipsi, $16.4 \mathrm{~ms} \pm 1.5 ;$ contra, $8.8 \mathrm{~ms}, \pm 1.6 ; p=0.026$, two-tailed $t$ test). HM, Horizontal meridian; VM, vertical meridian.

portions of area $8 \mathrm{Av}$, the region that projects directly to area MT (Petrides and Pandya, 2006).

\section{Mapping of lesion effects across the visual field}

Having established the nature of the deficit resulting from LPFC lesions, we examined whether the deficits for contralesional stimuli measured at specific visual field locations were uniformly distributed across the visual field by testing two of the monkeys (M123 and M908) at multiple locations within the central $20^{\circ}$. The mapping was performed with $1.5 \mathrm{~s}$ delay between $\mathrm{S} 1$ and S2. The data in Figure 4A,C illustrates the results of this mapping. The circles represent the size and the position of the visual stimuli used during mapping.
The values of average thresholds determined at each location are color-coded (blue-green, higher thresholds, yellow-red, lower thresholds). Within each hemifield, direction range thresholds were relatively unaffected by stimulus position and eccentricity, showing no consistent or systematic changes in thresholds with eccentricity. For example, in M123 the range thresholds measured at $3^{\circ}$ and $9^{\circ}$ eccentricity in the right hemifield were nearly identical $\left(330^{\circ}\right)$. This uniformity is reflected in the $\mathrm{SD}$ of the distribution of thresholds (SD: ipsi, $\pm 9^{\circ}$; contra, $\pm 11^{\circ}$ ) indicating that $66 \%$ of the measured thresholds was within $9^{\circ}-11^{\circ}$ of the mean. The range thresholds for M908 also showed relatively little variation with eccentricity within each hemifield (SD: ipsi, $\pm 14.5^{\circ}$; contra, $\left.\pm 8^{\circ}\right)$. The differences in thresholds emerged be- 
A

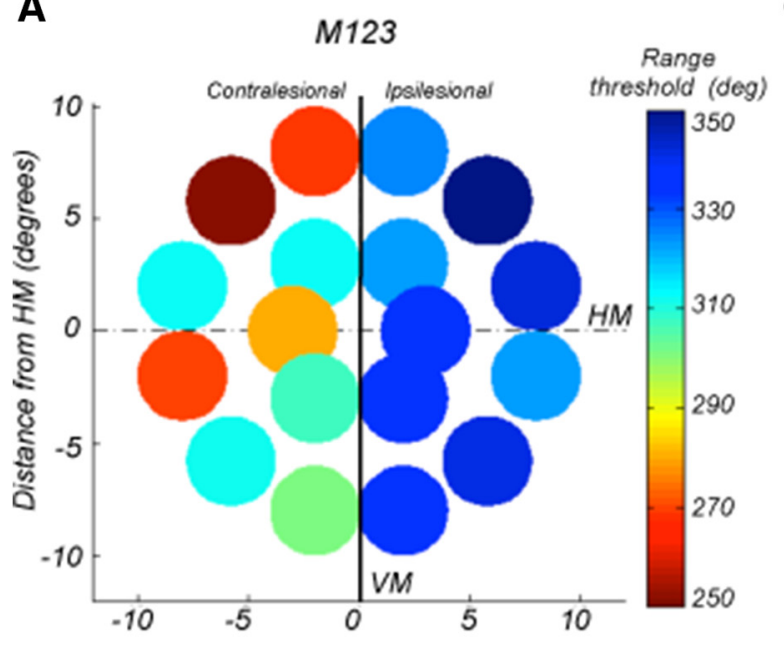

C

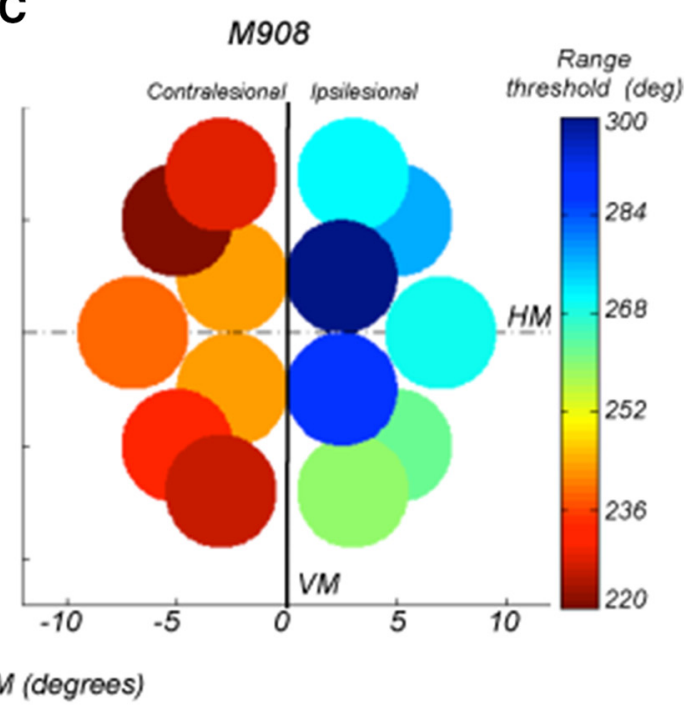

B

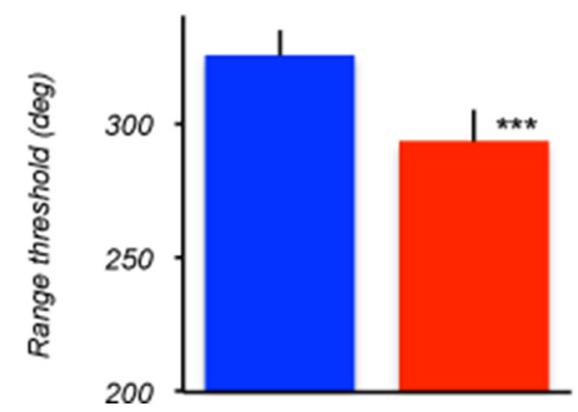

D

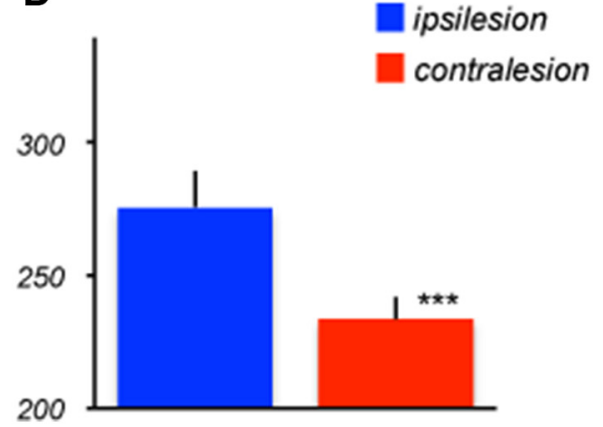

Figure 4. Mapping of direction range thresholds. $A, C$, Range thresholds for M123 and M908 measured at multiple locations in the ipsilesional and contralesional visual fields. Delay between S1 and $\mathrm{S2}: 1.5 \mathrm{~s}$. For M123, stimuli were $4^{\circ}$ diameter, moving at $15^{\circ} / \mathrm{s}$. For M908, stimuli were $5^{\circ}$ diameter, moving at $10^{\circ} \%$. Threshold values are color-coded (blue-green, higher thresholds, yellow-red, lower thresholds). Note, the superiority of nearly all thresholds measured in ipsilateral hemifields of both monkeys. $\boldsymbol{B}, \boldsymbol{D}$, Average range thresholds for M123 (B) and M908 (D) computed by combining all values measured within the ipsilesional (blue column) and contralesional (red column) hemifields. In both animals, contralesional thresholds were significantly poorer (M123 and M908, $p<0.000001$, two-tail $t$ test). Error bars are SD. HM, Horizontal meridian; VM, vertical meridian.

tween left and right hemifields (Figs. $4 B, D$ ), with poorer performance contralesional to the damage in the more posterior portions of LPFC. Monkey M123 showed consistently lower range thresholds for stimuli contralateral to the right brain hemisphere which sustained damage to areas $8 \mathrm{Ad}$ and $8 \mathrm{Av}$, than for stimuli contralateral to the unintended area 9/46d damage (Fig. $4 B ; p=8.4 \mathrm{e}^{-07}$; two-tailed $t$ test). Those thresholds were within the normal range determined for the intact monkey used in this study (Fig. 3C), as well as in other intact animals measured in our laboratory performing the same behavioral task (Bisley and Pasternak, 2000; Zaksas et al., 2001; Pasternak et al., 2003). It was also comparable, if not superior, to performance of the other two lesioned monkeys with stimuli in the ipsilesional hemifield. Thresholds in M908 were also inferior for the contralesional stimuli (Fig. $4 D ; p=3.8 \mathrm{e}^{-07}$; two-tailed $t$ test). The apparently intact thresholds, measured with stimuli contralateral to the hemisphere with damage to area $9 / 46 \mathrm{~d}$ and a small part of area $8 \mathrm{Ad}$, suggest that this region is unlikely to contribute to the tasks used in the current study. In both animals, the difference in performance between the two hemifields was of similar magnitude across the visual field. This uniformity suggests that signals residing in the PFC representing task relevant information are likely to be distributed across the contralesional visual field.
Motion signal thresholds are affected at a longer delay

Our previous work revealed that MT/MST lesion impaired direction thresholds measured by varying the coherence of random dots (Rudolph and Pasternak, 1999; Bisley and Pasternak, 2000). That version of the random dot stimulus consisted of coherently and randomly moving dots and its discriminability was manipulated by varying the proportion of coherently moving dots (percentage signal) during S1 (Pasternak et al., 1990; Pasternak and Merigan, 1994; Rudolph and Pasternak, 1999). With this stimulus, the deficit produced by MT/MST was present even at the shortest delay. We used that stimulus to determine whether the effects of LPFC damage were similar to those measured by manipulating the direction range. Signal thresholds were measured under two delay conditions, 0.25 and $1.5 \mathrm{~s}$. At $0.25 \mathrm{~s}$ delay, the thresholds of the three monkeys were similar for the two hemifields and ranged between $\sim 5 \%$ and 17\% (M123, $p=0.14 ; \mathrm{M} 601, p=0.4$; M908, $p=0.6, t$ test; Fig. 5). At the longer delay, however, all three animals showed degraded performance, and this degradation was greater for contralesional stimuli (M123, $p=$ 0.016; M601, $p=0.02$; M908, $p=0.035 ; t$ test), consistent with deficits for stimuli contralateral to the hemisphere with area $8 \mathrm{~A}$ damage revealed by measuring range thresholds. 
A

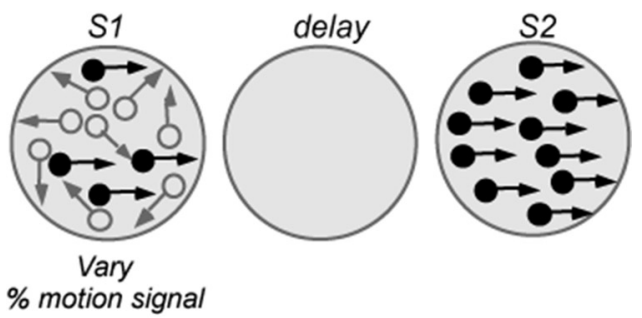

B
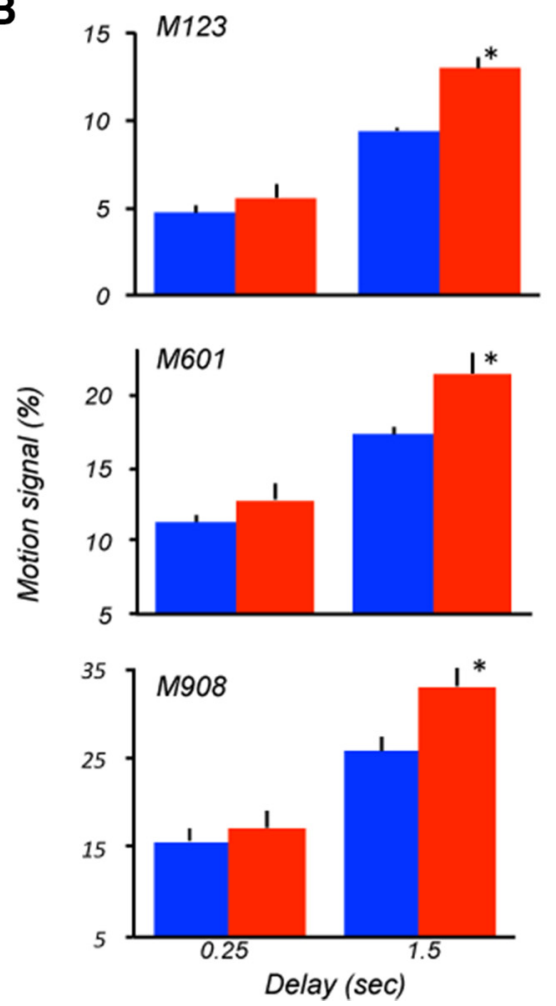

Figure 5. Lesion effects on motion signal thresholds. $\boldsymbol{A}$, Stimulus configuration used during the task. Thresholds for discriminating directions were measured by varying the proportion of dots moving in the same direction in the field of dots moving in random directions. Stimulus parameters and locations were identical to those used to measure range thresholds (Fig. 3). $\boldsymbol{B}$, Motion signal thresholds measured at short and longer delays for the three lesioned monkeys. At the shorter delay, all three animals showed similar ipsilesional and contralesional thresholds (M123, $p=0.12 ; \mathrm{M} 601, p=0.3 ; \mathrm{M} 908, p=0.61 ;$ two-tailed $t$ test). However, at the longer delay, all three monkeys showed weaker thresholds for contralesional stimuli (M123, $p=$ $0.008 ; \mathrm{M} 601, p=0.04 ; \mathrm{M} 908, p=0.014$ ).

In summary, the lesion effect measured with stimuli requiring extracting motion direction in the presence of noise was similar to that observed with stimuli requiring motion integration. At the shortest delay, contralesional, and ipsilesional motion-signal thresholds were similar, suggesting that the LPFC is unlikely to be necessary for processing of complex motion. However, the contralesional deficits at the longer delay provide additional support for the involvement of the LPFC in the ability to use directional signals retained during the delay.

\section{Delay-specific deficits do not depend on S1 coherence}

We also asked whether the observed deficits depended on the coherence of the stimuli signaling motion direction during S1. To address this question, in two of the animals (M123 and M908) we measured range thresholds by varying the direction range during S2, whereas the preceding S1 contained coherent motion (direc- tion range, $0^{\circ}$; Fig. $6 \mathrm{~A}$ ). The use of the same measure of performance in the two versions of the task allowed to directly compare monkey's ability to retain and use the information based on strong (suprathreshold) and weak (at threshold) motion information. As in the task where the direction range was varied during S1, the performance of both animals at the shortest delay was nearly identical for the two hemifields (Fig. 6B; M123, $p=0.9$; M908, $p=0.56$, two-tailed $t$ test). At longer delays, the thresholds decreased and this decrease was much greater for stimuli contralateral to the area that included area 8Av damage. ANCOVA of the whole dataset revealed a significant effect of delay $\left(F_{(1,41)}=\right.$ $\left.19 ; p=7.1 \mathrm{e}^{-05}\right)$, stimulus location $\left(F_{(1,43)}=15.3 ; p=0.00032\right)$, and a significant interaction between location and delay $\left(F_{(1,43)}=\right.$ $8.5 ; p=0.0056)$, again confirming that the degradation in performance with increasing delay was greater in the contralesional hemifield.

It is noteworthy that ipsilesional thresholds measured with S1 containing coherent motion showed a very modest change with increasing delay ( $\operatorname{tau}=51 \pm 1)$, compared with that measured with $\mathrm{S} 1$ at range threshold $(\mathrm{tau}=16.4 \pm 1.5)$. This slow decay of memory for coherent motion was also observed in the earlier study (Bisley and Pasternak, 2000). In contrast, the contralesional thresholds decreased rapidly with delay and this decrease was nearly identical to that measured when S1 contained the broad range of directions (Fig. $3 C$ ). Thus, the deficit with increasing delays produced by the LPFC damage did not depend on the type of motion that gave rise to the remembered direction.

\section{PFC damage degrades the ability to rapidly shift spatial attention}

In the experiments described above, the two comparison stimuli always appeared at the same, highly predictable location. Under these conditions, prefrontal damage produced moderate deficits in the ability to retain and use information about contralesional motion direction. We examined whether these deficits would be exacerbated if the location of the comparison S2 was unpredictable. To successfully compare directions of S1 and S2, the animal must be able to rapidly shift attention between widely separated spatial locations (Fig. 7A). We measured range thresholds in the ipsilesional and contralesional hemifields, with S1 always appearing at the same predictable location, while the location of S2 was either highly predictable or uncertain. When the S2 location was predictable, within the same block of trials it appeared $12^{\circ}$ (M601), $16^{\circ}(\mathrm{M} 123)$, or $5^{\circ}$ (M908) away from S1 within the same hemifield. However, when the location of S2 was uncertain, it appeared with $50 \%$ probability either at the location of S1 or at a distant location. To ensure that we were comparing performance of stimuli in the same spatial configuration, in the uncertain condition, we only analyzed trials where S1 and S2 were in different locations, but these trials were randomly interleaved with trials with $S 1$ and S2 appearing in the same location. In this version of the task, $\mathrm{S} 1$ and $\mathrm{S} 2$ were separated by a $1.5 \mathrm{~s}$ delay and we varied the range of $S 1$, whereas $S 2$ was always presented at the $0^{\circ}$ range. Figure $7 B$ provides the comparison of performance in the ipsilesional and contralesional hemifields of the three lesioned animals when the location of S2 was predictable and unpredictable.

In the ipsilesional hemifield, thresholds were largely unaffected by location uncertainty (M123, $p=0.16$; M601, $p=0.45$; M908, $p=0.8$; $t$ test). However, in the contralesional hemifield, location uncertainty affected the range thresholds (M123, $p=$ 0.006; M601, $p=0.045$; M908, $p=0.017$; $t$ test). The effect of spatial uncertainty of $S 2$ placed within the ipsilateral and contralesional hemifield was also assessed by pooling the data from the 
three animals. The Welch two-sample $t$ test showed a significant effect of uncertainty for the contralesional $(p=$ $0.00015)$ but not for the ipsilesional hemifield $(p=0.126)$. These results show that the uncertainty about the location of the comparison stimulus presents an additional challenge to subjects with prefrontal damage. Most likely, this challenge is introduced by the need to rapidly shift attention to the quadrant containing the comparison stimulus. It is noteworthy that the effect was as pronounced in the monkey with the small circumscribed lesion concentrated on the more ventral portion of area 8A (M601), as in the animals with more extensive damage, suggesting a role for the more ventral portions of $8 \mathrm{~A}$ involved in the lesions in all three monkeys. The detrimental effect of LPFC damage on the ability to rapidly shift spatial attention during the delayed direction discrimination task highlights a role of the affected LPFC region in the attentional aspects of such tasks.

\section{Discussion}

We examined the effects of unilateral lesions of LPFC to the maintenance and utilization of visual motion during a working memory task. We found the deficits in the ability to compare current and remembered directions of motion presented in the contralesional hemifield. These deficits appeared only at longer delays, were of similar magnitude across the contralesional visual field, and did not depend on motion coherence. In addition, lesion effects were most pronounced when the comparison stimulus S2 appeared in an unpredictable visual field location, the condition that required rapid shifts of attention.

\section{Contralesional effects suggest interactions with extrastriate visual cortex}

Our results show that the LPFC damage only affected the performance with stimuli contralateral to the hemisphere with damage that included areas 8Av and 8Ad. Careful mapping revealed deficits across multiple locations of the contralesional hemifield, with the vertical meridian providing clear separation between the intact and reduced performance. This pattern of results points to the importance of connectivity between the LPFC and the retinotopically organized cortical areas that process contralateral visual stimuli within the same hemisphere (Barbas, 1988; Schall et al., 1995; Petrides and Pandya, 2006; Ninomiya et al., 2012). It is also consistent with reports of the preference in the prefrontal cortex for contralateral stimuli (Boch and Goldberg, 1989; Funahashi et al., 1989; Sakagami and Niki, 1994; Rainer et al., 1999; Everling et al., 2002).

Deficits confined to the contralesional hemifield have been observed in patients with unilateral prefrontal damage tested on a

C
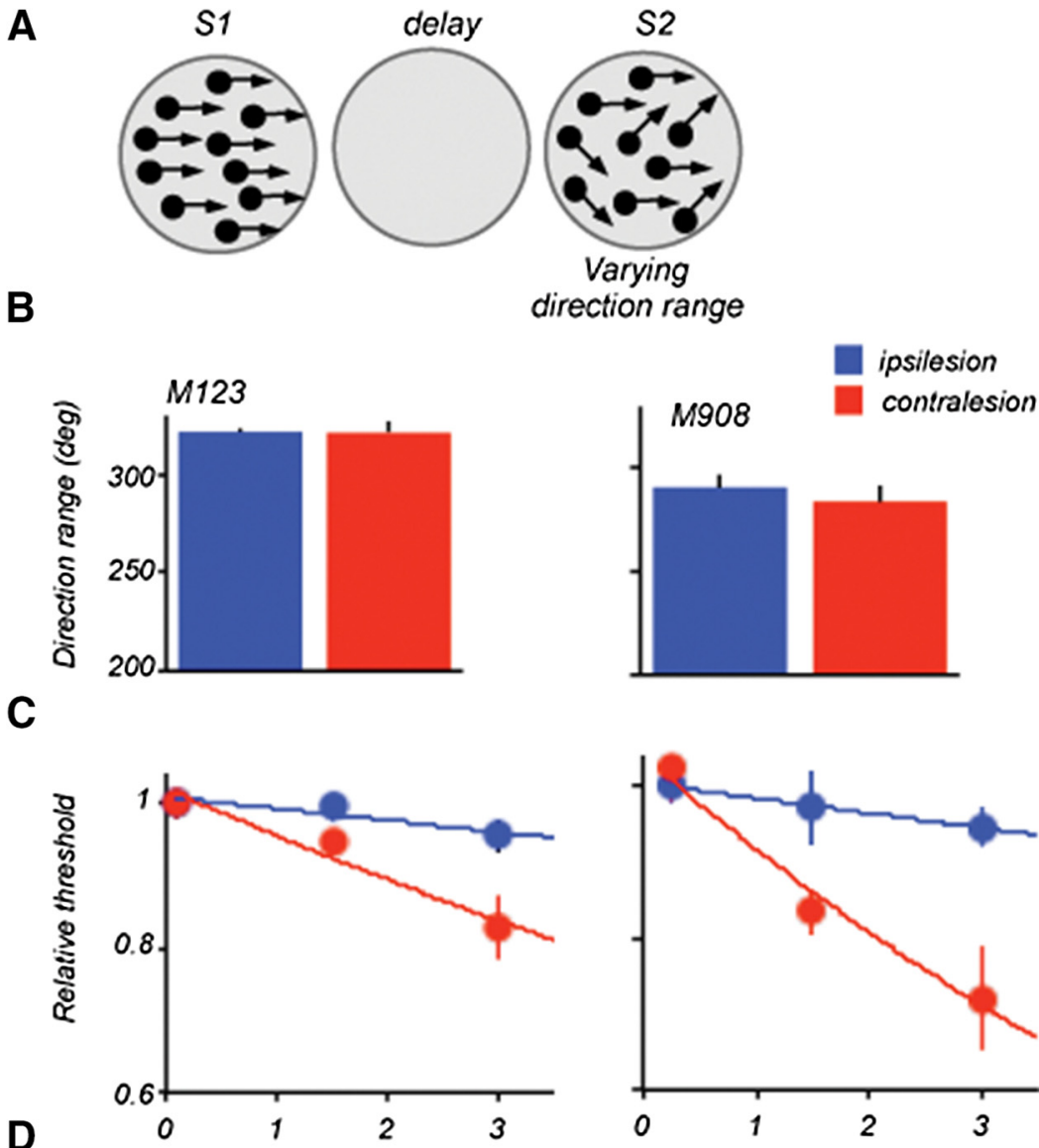

Delay (sec)

Figure 6. Lesion effects on direction range thresholds: $\mathrm{S} 1$ at the $0^{\circ}$ range. $\boldsymbol{A}$, Stimulus configuration used during the task Thresholds were measured by varying direction range during S2. $\boldsymbol{B}$, Range thresholds measured at the shortest delay in M123 and ds measured at the shortest delay and fitted with an exponential function. D. Comparison of average decay times (tau) computed by fitting an exponential to the ipsilesional and contralesional data for the two lesioned monkeys. The estimated decay time was shorter for the contralesional dataset (ipsi, $51 \mathrm{~ms} \pm 1.3$; contra, $10.6 \mathrm{~ms}, \pm 2.7 ; p=0.005$, two-tailed $t$ test).

visual working memory task (Voytek and Knight, 2010; Voytek et al., 2010). These studies also reported weakening in attentionrelated event-related potentials (ERPs) recorded during the working memory task, the result interpreted as a loss of top-down control of visual attention in extrastriate cortex within the same hemisphere. Contralesional deficits and abnormal ERPs in extrastriate cortex have also been seen in patients with unilateral dorsolateral prefrontal damage during a visual attention task (Barceló et al., 2000).

\section{LPFC and motion processing}

We previously reported that during motion comparison tasks, neurons in the LPFC show responses to reminiscent of activity recorded in area MT (Zaksas and Pasternak, 2006; Hussar and Pasternak, 2009, 2012, 2013). Selectivity of these neurons for direction has also been observed during other tasks involving visual 
A

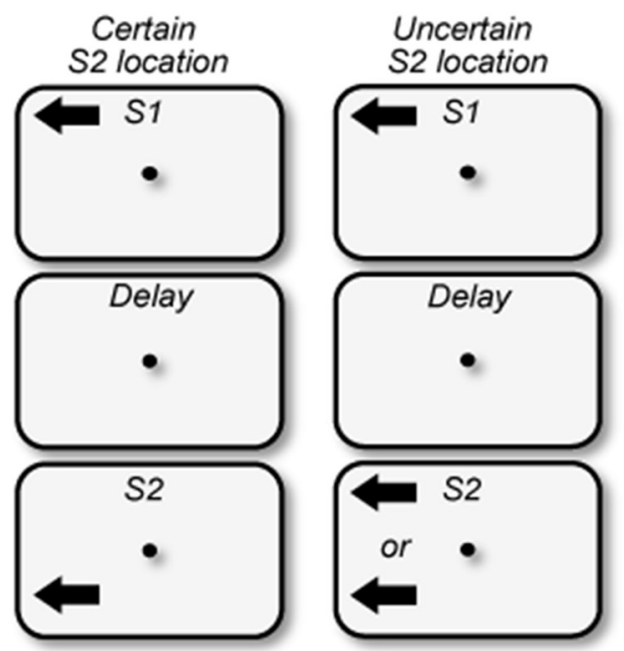

B

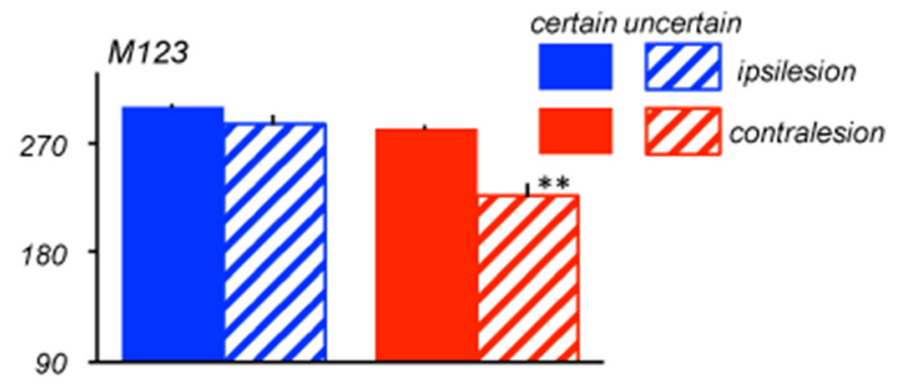

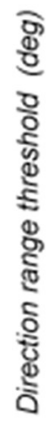
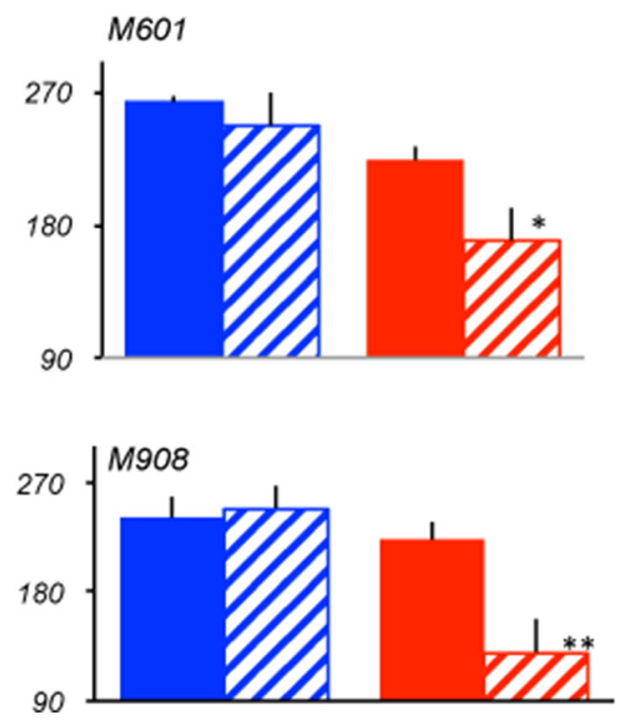

Figure 7. Effects of location uncertainty on ipsilesional and contralesional range thresholds. A, Stimulus configuration during certain (left) and uncertain (right) locations of S2. Stimulus speed: $15 \%$ s (M123 and M601); 10\% (M908). On trials with certain $\mathrm{S} 2$ locations, $\mathrm{S} 1$ and $\mathrm{S} 2$ always appeared at the same locations within the same hemifield and were separated by $16^{\circ}\left(\mathrm{M} 123 ; \mathrm{S} 1: \pm 8^{\circ} / 8^{\circ} ; \mathrm{S} 2 \pm 8^{\circ} /-8^{\circ}\right), 12 \mathrm{deg}\left(\mathrm{M} 601 ; \mathrm{S} 1: \pm 6^{\circ} / 6^{\circ} ; \mathrm{S} 2 \pm 6^{\circ} \%-6^{\circ}\right)$ and $5^{\circ}\left(\mathrm{M} 908 ; \mathrm{S} 1: \pm 5^{\circ} / 5^{\circ} ; \mathrm{S} 2 \pm\right.$ $\left.5^{\circ} / 0^{\circ}\right)$. On trials with uncertain locations of $\mathrm{S} 2, \mathrm{~S} 1$ always appeared at the same location in the upper quadrant, while $\mathrm{S} 2$ appeared with $50 \%$ probability at the same location as $S 1$ or in a separate location within the same hemifield. On trials with separated S1 and S2, the distance between them was identical to trials with certain S2 locations (see above). All thresholds were measured with $1.5 \mathrm{~s}$ delay between $\mathrm{S1}$ and $\mathrm{S2}$. B, Ipsilesional (blue) and contralesional (red) range thresholds measured in blocks of trials with certain (solid) and uncertain (striped) locations of $\mathrm{S} 2$. All three animals showed no significant difference in performance between certain and uncertain blocks of trials with ipsilesional stimuli (M123, $p=$ $0.16 ; \mathrm{M} 601, p=0.26 ; \mathrm{M} 908, p=0.8$; two-tailed $t$ test). However, in the contralesional hemifield, the range thresholds were poorer when the location of $S 2$ was uncertain (M123, $p=0.006 ; M 601, p=0.045 ;$ M908, $p=0.018$; two-tailed $t$ test). The Welch two-sample $t$ test for the data pooled for the three monkeys also showed a significant effect of uncertainty for the contralesional ( $p=0.00015$ ) but not for the ipsilesional hemifield ( $p=0.126$ ). motion (Kim and Shadlen, 1999; Swaminathan and Freedman, 2012; MendozaHalliday et al., 2014). The presence of direction selectivity in LPFC and its reciprocal connections with areas MT and MST raises the possibility of a role it may play in sensory components of the task. The data collected on trials with the shortest delays (100-250 ms) allowed us to focus on motion processing during the task, while placing minimal demands on its nonsensory aspects. We found that neither the range nor motion signals thresholds were affected by the lesion, suggesting that the LPFC is unlikely to play a major role in the integration of local motion into a global signal or in the extraction of coherent motion from noise, two operations required by our tasks. Thus, despite the presence of detailed information about visual motion (Hussar and Pasternak, 2013), LPFC does not appear to be necessary for the successful implementation of the sensory task components. This finding is in contrast to the deficits in motion thresholds at short delays produced by the MT/MST lesions during the same task (Rudolph and Pasternak, 1999), further confirming the distinct roles of the LPFC and MT in motion discriminations.

\section{LPFC and MT/MST lesion effects are different at longer delays}

The distinct contributions of LPFC and MT to memory-guided motion comparisons are also exemplified by differences in lesion effects at longer delays. Like the unilateral damage of LPFC, unilateral MT/MST lesion produced contralesional deficit in range thresholds that increased with delay (Bisley and Pasternak, 2000). However, this deficit was only present when the remembered stimulus contained the broad range of directions and required motion integration, the process to which MT neurons uniquely contribute (Movshon et al., 1985; Bisley et al., 2004). In contrast, when the remembered motion was coherent, the performance at longer delays was unaffected by the lesion, suggesting that this region was not critical for the preservation of stimuli that did not require motion integration. This pattern of results led to the conclusion that MT neurons contribute to remembering stimuli they process (Bisley and Pasternak, 2000; Pasternak and Greenlee, 2005) and linked MT to the circuitry involved in the storage and utilization of visual motion during comparison tasks. Participation of area MT in this circuitry received further support from the fact that microstimulation of identified sites in MT applied during the delay severely disrupted dis- 
crimination of directions in the contralateral hemifield (Bisley et al., 2001). Subsequent recording studies revealed activity in MT consistent with its participation in the circuitry supporting memory-guided comparisons (Bisley et al., 2004; Zaksas and Pasternak, 2006; Lui and Pasternak, 2011).

The damage to LPFC, like the damage to MT/MST, also produced delay-specific impairment of motion comparisons. However, unlike the effect produced by the MT/MST lesion, this deficit was as pronounced when the remembered S1 was coherent as when it was at threshold. Thus, although the contribution of MT/MST to the task strongly depended on stimuli generating the remembered direction, the LPFC lesion effect was not linked to the nature of the remembered stimulus. This observation suggests that the role of LPFC in sensory maintenance may be in accessing and utilization of the preserved information, rather the actual storage of signals giving rise to the remembered direction.

This interpretation of the LPFC lesion effects is consistent with the proposal that the role of prefrontal cortex in the maintenance of relevant sensory information lies in directing attention to its representation stored elsewhere, and in manipulation and assistance in selecting appropriate action (Curtis and D'Esposito, 2003; Szczepanski and Knight, 2014). The role of prefrontal cortex in monitoring and manipulating information during working memory tasks has also been highlighted by the work of Petrides (2000).

Attentional abnormalities in human patients with unilateral prefrontal damage performing a working memory task provide further evidence in favor of this interpretation (Voytek and Knight, 2010). A role for prefrontal cortex in controlling visual attention was also documented in a study showing that relatively modest deficits in orientation discrimination in monkeys with a unilateral prefrontal lesion dramatically increased when the task required attentional shifts across trials (Rossi et al., 2007). These behavioral observations received recent physiological support showing reduced attentional effects in area V4 in animals with unilateral PFC lesions in the same hemisphere (Gregoriou et al., 2014). Our finding of impaired thresholds on trials requiring rapid shifts of attention is consistent with these results, strengthening the notion of the key role played by LPFC in the allocation of attention to sensory stimuli.

One of the observations in this and other studies involving permanent unilateral PFC damage is relatively modest size of the effects. This is apparent in both humans (Corbetta et al., 2005) and nonhuman primates (Rossi et al., 2007; Gregoriou et al., 2014). This partial preservation of function is often attributed to compensatory processes and reorganization of relevant circuits (Corbetta et al., 2005). There is evidence that such processes can be rapid, involving the activation of the intact PFC of the opposite hemisphere (Voytek et al., 2010). In the present study, the substantial sparing of the ability of perform memory-guided motion comparisons may be a reflection of compensatory changes that may have occurred during the course of postlesion testing. However, in many cases, the deficits measured even during the first postlesion sessions were at levels comparable with those obtained at the end of data collection, suggesting either rapid compensatory changes involving the intact hemisphere and/or incomplete damage of subregions within the LPFC relevant to the task. This possibility is supported by the deficit observed in the monkey M601 with the smallest lesion that included area $8 \mathrm{Av}$, the region reciprocally interconnected with area MT. Future studies involving reversible inactivation of specific subregions within the LFPC during behavioral testing will help to determine whether compensatory changes and/or the location and extent of the lesion are responsible for the partially preserved ability to perform memory-guided motion discriminations.

\section{Lesion location and behavioral effects}

Because of the small number of cases in the present study we were not able to systematically explore the relationship between behavioral effects and the extent of the damage. Nevertheless, our results point to the importance of specific portions of the damaged cortex in determining the observed sensory deficits. This is particularly well documented in M123, who sustained damage in both brain hemispheres. In the hemifield contralateral to the unintended damage to area $9 / 46 \mathrm{~d}$ and a portion of area $8 \mathrm{Ad}$, the performance was within the normal range on all behavioral tasks. One possible reason for the intact performance with stimuli contralateral to the damaged area 9/46 is the nonspatial nature of our task, since this portion of the prefrontal cortex has been shown to represent spatial information (Funahashi, 2013) and has been strongly linked to spatial working memory (Goldman-Rakic, 1996). In contrast to the intact thresholds for stimuli contralateral to the damaged area $9 / 46 \mathrm{~d}$, this animal showed deficits on all tasks with stimuli contralateral to the damaged areas within area $8 \mathrm{~A}$ (8Av and $8 \mathrm{Ad}$ ). Thus, the most likely contributor to the loss of performance at longer delays was the damage to area $8 \mathrm{Av}$. This is further supported by the fact that although in M601 the lesion was limited primarily to the ventral portion of area $8 \mathrm{~A}(8 \mathrm{Av})$, M908 sustained more extensive damage that included areas $8 \mathrm{Ad}$ and $8 \mathrm{Av}$, as well as the tissue within and around the upper and lower limbs of the arcuate. Despite these differences, the pattern of functional loss in the three animals was similar, implicating areas $8 \mathrm{Ad}$ and especially $8 \mathrm{Av}$ in the observed effects. In addition to the damage in the prearcuate region involving area $8 \mathrm{~A}, \mathrm{M} 123$ also sustained damage to the dorsal premotor cortex. This additional damage was unlikely to have contributed to the deficits seen here, because the loss displayed by this monkey was relatively modest and similar to that of the other two animals that did not sustain damage to the dorsal premotor cortex. Overall, the similarity in the pattern of deficits observed in the three monkeys and the involvement of area $8 \mathrm{Av}$ in their lesions suggests a role of this portion of prefrontal cortex in motion comparisons. In the context of our task, area $8 \mathrm{Av}$ is of particular interest because of its direct reciprocal connectivity with motion processing areas MT and MST (Petrides and Pandya, 2006; Ninomiya et al., 2012). Although in all animals the lesion extended into area $8 \mathrm{Av}$, the damage to this area was less extensive in M123, and this monkey exhibited relatively modest deficits. This apparent link between damage to area $8 \mathrm{Av}$ and motion discrimination deficits requires further confirmation. Nevertheless, it is likely that the deficit on tasks requiring retention and comparison of motion information is associated with the disruption of top-down influences arriving from the PFC in motion processing cortical areas.

In conclusion, our study revealed the contribution of the LPFC neurons to tasks requiring comparisons between the current and remembered contralateral stimuli separated in time. Although this contribution reflects the interaction with visual neurons processing contralateral motion, it is independent of specific stimulus features giving rise to the information about motion direction. These results demonstrate the importance of the LPFC to the maintenance stage of the motion comparison task. However, they argue against LPFC being the site of storage of the original motion signals giving rise to directions used during the task. Rather these results support its role in accessing such signals during the delay and allowing their utilization in the comparison process. 


\section{References}

Barbas H (1988) Anatomic organization of basoventral and mediodorsal visual recipient prefrontal regions in the rhesus monkey. J Comp Neurol 276:313-342. CrossRef Medline

Barceló F, Suwazono S, Knight RT (2000) Prefrontal modulation of visual processing in humans. Nat Neurosci 3:399-403. CrossRef Medline

Bartus RT, Levere TE (1977) Frontal decortication in rhesus monkeys: a test of the interference hypothesis. Brain Res 119:233-248. CrossRef Medline

Bisley JW, Pasternak T (2000) The multiple roles of visual cortical areas $\mathrm{MT} / \mathrm{MST}$ in remembering the direction of visual motion. Cereb Cortex 10:1053-1065. CrossRef Medline

Bisley JW, Zaksas D, Pasternak T (2001) Microstimulation of cortical area MT affects performance on a visual working memory task. J Neurophysiol 85:187-196. Medline

Bisley JW, Zaksas D, Droll JA, Pasternak T (2004) Activity of neurons in cortical area MT during a memory for motion task. J Neurophysiol 91: 286-300. CrossRef Medline

Boch RA, Goldberg ME (1989) Participation of prefrontal neurons in the preparation of visually guided eye movements in the rhesus monkey. J Neurophysiol 61:1064-1084. Medline

Corbetta M, Kincade MJ, Lewis C, Snyder AZ, Sapir A (2005) Neural basis and recovery of spatial attention deficits in spatial neglect. Nat Neurosci 8:1603-1610. CrossRef Medline

Curtis CE, D'Esposito M (2003) Persistent activity in the prefrontal cortex during working memory. Trends Cogn Sci 7:415-423. CrossRef Medline

D’Esposito M, Postle BR (2015) The cognitive neuroscience of working memory. Annu Rev Psychol 66:115-142. CrossRef Medline

Everling S, Tinsley CJ, Gaffan D, Duncan J (2002) Filtering of neural signals by focused attention in the monkey prefrontal cortex. Nat Neurosci 5:671-676. CrossRef Medline

Funahashi S (2013) Space representation in the prefrontal cortex. Prog Neurobiol 103:131-155. CrossRef Medline

Funahashi S, Bruce CJ, Goldman-Rakic PS (1989) Mnemonic coding of visual space in the monkey's dorsolateral prefrontal cortex. J Neurophysiol 61:331-349. Medline

Funahashi S, Bruce CJ, Goldman-Rakic PS (1993) Dorsolateral prefrontal lesions and oculomotor delayed-response performance: evidence for mnemonic "scotomas". J Neurosci 13:1479-1497. Medline

Fuster JM, Alexander GE (1971) Neuron activity related to short-term memory. Science 173:652-654. CrossRef Medline

Goldman-Rakic PS (1995) Cellular basis of working memory. Neuron 14: 477-485. CrossRef Medline

Goldman-Rakic PS (1996) Regional and cellular fractionation of working memory. Proc Natl Acad Sci U S A 93:13473-13480. CrossRef Medline

Gregoriou GG, Rossi AF, Ungerleider LG, Desimone R (2014) Lesions of prefrontal cortex reduce attentional modulation of neuronal responses and synchrony in V4. Nat Neurosci 17:1003-1011. CrossRef Medline

Hussar CR, Pasternak T (2009) Flexibility of sensory representations in prefrontal cortex depends on cell type. Neuron 64:730-743. CrossRef Medline

Hussar C, Pasternak T (2010) Trial-to-trial variability of the prefrontal neurons reveals the nature of their engagement in a motion discrimination task. Proc Natl Acad Sci U S A 107:21842-21847. CrossRef Medline

Hussar CR, Pasternak T (2012) Memory-guided sensory comparisons in the prefrontal cortex: contribution of putative pyramidal cells and interneurons. J Neurosci 32:2747-2761. CrossRef Medline

Hussar CR, Pasternak T (2013) Common rules guide comparisons of speed and direction of motion in the dorsolateral prefrontal cortex. J Neurosci 33:972-986. CrossRef Medline

Jacobsen CF (1936) The functions of the frontal association areas in monkeys. Comp Psychol Monographs 13:1-60.

Kim JN, Shadlen MN (1999) Neural correlates of a decision in the dorsolateral prefrontal cortex of the macaque. Nat Neurosci 2:176-185. CrossRef Medline

Kutner M, Nachtsheim CJ, Neter J, Li W (2004) Applied linear statistical models. New York: McGraw-Hill/Irwin.

Lebedev MA, Messinger A, Kralik JD, Wise SP (2004) Representation of attended versus remembered locations in prefrontal cortex. PLoS Biol 2:e365. CrossRef Medline

Lui LL, Pasternak T (2011) Representation of comparison signals in cortical area MT during a delayed direction discrimination task. J Neurophysiol 106:1260-1273. CrossRef Medline

Malmo RB (1942) Interference factors in delayed response in monkey after removal of the frontal lobes. J Neurophysiol 5:295-308.
Mendoza-Halliday D, Torres S, Martinez-Trujillo JC (2014) Sharp emergence of feature-selective sustained activity along the dorsal visual pathway. Nat Neurosci 17:1255-1262. CrossRef Medline

Miller EK, Buschman TJ (2013) Cortical circuits for the control of attention. Curr Opin Neurobiol 23:216-222. CrossRef Medline

Miller EK, Cohen JD (2001) An integrative theory of prefrontal cortex function. Annu Rev Neurosci 24:167-202. CrossRef Medline

Movshon JA, Adelson EH, Gizzi MS, Newsome WT (1985) The analysis of moving visual patterns. In: Pattern recognition mechanisms (Chagas C, Gattas R, Gross CG, eds), pp 117-151. New York: Springer.

Ninomiya T, Sawamura H, Inoue K, Takada M (2012) Segregated pathways carrying frontally derived top-Down signals to visual areas MT and V4 in macaques. J Neurosci 32:6851-6858. CrossRef Medline

Pasternak T, Greenlee MW (2005) Working memory in primate sensory systems. Nat Rev Neurosci 6:97-107. CrossRef Medline

Pasternak T, Merigan WH (1994) Motion perception following lesions of the superior temporal sulcus in the monkey. Cereb Cortex 4:247-259. CrossRef Medline

Pasternak T, Albano JE, Harvitt DM (1990) The role of directionally selective neurons in the perception of global motion. J Neurosci 10:30793086. Medline

Pasternak T, Bisley JW, Calkins D (2003) Visual information processing in the primate brain. In: Biological psychology (Michaela Gallagher, Nelson RJ, eds), pp 139-185. New York: Wiley.

Paxinos G, Huang XF, Toga A (2000) The rhesus monkey brain in stereotaxic coordinates. San Diego: Academic.

Petrides M (2000) The role of the mid-dorsolateral prefrontal cortex in working memory. Exp Brain Res 133:44-54. CrossRef Medline

Petrides M, Pandya DN (2006) Efferent association pathways originating in the caudal prefrontal cortex in the macaque monkey. J Comp Neurol 498:227-251. CrossRef Medline

Quick RF Jr (1974) A vector-magnitude model of contrast detection. Kybernetik 16:65-67. CrossRef Medline

Rainer G, Rao SC, Miller EK (1999) Prospective coding for objects in primate prefrontal cortex. J Neurosci 19:5493-5505. Medline

Rossi AF, Bichot NP, Desimone R, Ungerleider LG (2007) Top down attentional deficits in macaques with lesions of lateral prefrontal cortex. J Neurosci 27:11306-11314. CrossRef Medline

Rudolph K, Pasternak T (1999) Transient and permanent deficits in motion perception after lesions of cortical areas MT and MST in the macaque monkey. Cereb Cortex 9:90-100. CrossRef Medline

Sakagami M, Niki H (1994) Spatial selectivity of go/no-go neurons in monkey prefrontal cortex. Exp Brain Res 100:165-169. Medline

Schall JD, Morel A, King DJ, Bullier J (1995) Topography of visual cortex connections with frontal eye field in macaque: convergence and segregation of processing streams. J Neurosci 15:4464-4487. Medline

Sreenivasan KK, Curtis CE, D'Esposito M (2014) Revisiting the role of persistent neural activity during working memory. Trends Cogn Sci 18:82-89. CrossRef Medline

Swaminathan SK, Freedman DJ (2012) Preferential encoding of visual categories in parietal cortex compared with prefrontal cortex. Nat Neurosci 15:315-320. CrossRef Medline

Szczepanski SM, Knight RT (2014) Insights into human behavior from lesions to the prefrontal cortex. Neuron 83:1002-1018. CrossRef Medline

Tsujimoto S, Postle BR (2012) The prefrontal cortex and oculomotor delayed response: A reconsideration of the "mnemonic scotoma." J Cogn Neurosci 24:627-635. CrossRef Medline

Voytek B, Knight RT (2010) Prefrontal cortex and basal ganglia contributions to visual working memory. Proc Natl Acad Sci U S A 107:1816718172. CrossRef Medline

Voytek B, Davis M, Yago E, Barceló F, Vogel EK, Knight RT (2010) Dynamic neuroplasticity after human prefrontal cortex damage. Neuron 68:401408. CrossRef Medline

Wong-Riley M (1979) Changes in the visual system of monocularly sutured or enucleated cats demonstrable with cytochrome oxidase histochemistry. Brain Res 171:11-28. CrossRef Medline

Zaksas D, Pasternak T (2006) Directional signals in the prefrontal cortex and in area MT during a working memory for visual motion task. J Neurosci 26:11726-11742. CrossRef Medline

Zaksas D, Bisley JW, Pasternak T (2001) Motion information is spatially localized in a visual working-memory task. J Neurophysiol 86:912-921. Medline 